\title{
Ein Instrument für die Beratung bei vorgeburtlichen Untersuchungen
}

Rosmarie Pinzello-Hürlimann

Dr. med., Fachärztin für Allgemeinmedizin mit Schwerpunkt Frauenmedizin und Schwangerschaftsbetreuung

Interessenbindungen: Die Autorin ist Vorstandsmitglied des Vereins für ganzheitliche Beratung in der pränatalen Diagnostik, der Mitherausgeber der vorgestellten Broschüre ist.

Korrespondenz: Dr. med. Rosmarie PinzelloHürlimann

FMH Allgemeine Medizin

Grabengasse 12

CH-8180 Bülach
Der Leitfaden «Psychosoziale Beratung bei vorgeburtlichen Untersuchungen» (Anleitungen für die Beratungspraxis ergänzt mit interdisziplinärem Fachwissen), der für Schwangerschafts- und Familienberatungsstellen erarbeitet worden ist, ist auch für Ärztinnen und Ärzte ein wertvolles Beratungsinstrument. Gemeinsam entwickelt und herausgegeben haben das neue Beratungsinstrument das Interdisziplinäre Institut für Ethik im Gesundheitswesen der Stiftung Dialog Ethik; PlanEs, Schweizerische Stiftung für sexuelle und reproduktive Gesundheit; VSSB, Verband der Schwangerschafts- und Sexualberaterinnen; Verein Ganzheitliche Beratung und kritische Information zu pränataler Diagnostik.

Pränatale Untersuchungen haben das Ziel, Risiken für Mutter und Kind rechtzeitig zu erkennen, eine optimale Behandlung zu gewährleisten und dabei Ängste der Eltern abzubauen. Die Entwicklungen in der pränatalen Diagnostik (PND) haben in den letzten Jahren zu einer starken Ausweitung der vorgeburtlichen Untersuchungen auf Frauen und Paare ohne besonderes Risiko geführt.

Diese Entwicklungen wirken sich auch auf die Beratungspraxis der Ärzteschaft aus und werden zunehmend zu einer Herausforderung: Einerseits hat man die Pflicht als Ärztin/Arzt, jede Patientin schon sehr früh in der Schwangerschaft kompetent und ausführlich über Vor- und Nachteile der PND zu informieren und verschiedene Tests anzubieten (unter Berücksichtigung des Rechts auf Nichtwissen; Art. 13 der Krankenpflege-Leistungsverordnung KLV), anderseits will man die Patientin nicht unnötig verunsichern.

In welchem Mass darf der Arzt Entscheidungsempfehlungen aussprechen, in welchem Mass muss er sich nicht-direktiv verhalten? Unter zunehmendem juristischem Druck besteht die Gefahr, dass das ärztliche Aufklärungsgespräch einseitig von haftungsrechtlichen Gesichtspunkten geprägt wird, die medizinischen und menschlichen Aspekte dagegen

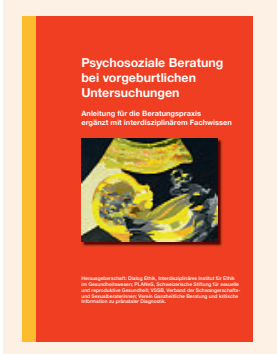

Dialog Ethik et al. (Hrsg.) Psychosoziale Beratung bei vorgeburtlichen Untersuchungen

Anleitung für die Beratungspraxis ergänzt mit interdisziplinärem Fachwissen Zürich: Dialog Ethik; 2010. $49 \mathrm{CHF}$

ISBN 978-3-905757-02-6 zurückgedrängt werden und so die vertrauensvolle Arzt-Patientinnen-Beziehung beeinträchtigt wird. Dies ist gerade deshalb problematisch, weil im Rahmen vorgeburtlicher Untersuchungen für Ärztin, Arzt und schwangere Frau komplexe ethische Dilemma-Situationen entstehen können, die ganz im Interesse der Frau und des werdenden Lebens anzugehen sind. Diese Beratungstätigkeit nimmt in der Sprechstunde sehr viel Zeit in Anspruch und fordert die Kompetenz des Arztes in vielen Bereichen.

Jeder Arzt, der Ultraschalluntersuchungen in der Schwangerschaft durchführt, muss einen Kurs in «kommunikativer Kompetenz» von drei Stunden absolvieren. Obwohl diese Massnahme sehr umstritten ist, ist sie doch eine Chance, die Ärzte für die vielfältigen Fragen rund um die pränatale Diagnostik zu sensibilisieren und die Beratung in der PND zu verbessern. Der sehr ausführliche Leitfaden ist als Unterstützung für diese Kurse sehr geeignet, zumal er das Grundwissen in der pränatalen Diagnostik auffrischt und das ärztliche Wissen mit Informationen aus den Gebieten von Ethik, Recht und Heilpädagogik ergänzt.

Besonders wertvoll für den Gesprächsablauf sind die Checklisten. Die Strukturierung des Beratungsablaufs ist eine Hilfe in dem von Zeitdruck geprägten Praxisalltag. Sehr hilfreich sind auch die Listen mit Links zu den verschiedenen Institutionen und Beratungsstellen sowie die Literaturangaben.

Die Beratung soll Paare nicht nur über die Möglichkeiten der pränatalen Diagnostik informieren, sondern unsere Patientinnen und ihre Partner bei ihrem persönlichen Entscheidungsfindungsprozess begleiten; sei es, dass sie sich für das Austragen eines Kindes mit einer Abweichung oder für einen Schwangerschaftsabbruch entscheiden. In jedem Fall sollen sie auch an entsprechende Organisationen verwiesen werden können, die ihnen über die ärztliche Beratung hinaus die nötige Unterstützung bieten. Es ist wichtig, dass auch der begleitende Arzt oder die begleitende Ärztin offen sind für die Geburt eines Kindes mit einer Abweichung und sich zusammen mit dem Paar auch auf eine solche Geburt einstellen und freuen können.

Fortbildungshinweis: Beratung bei vorgeburtlichen Untersuchungen unter besonderer Berücksichtigung ethischer, rechtlicher und psychosozialer Aspekte. Donnerstag, 8. September 2011, 13.30-17.45 Uhr. Anmeldung: info@ dialog-ethik.ch 